\title{
MULTIPLE FATIGUE CRACK GROWTH MODELLING IN NONHOMOGENEOUS STRUCTURAL SYSTEMS USING THE DUAL BEM
}

\author{
HEIDER DE CASTRO E ANDRADE \& EDSON DENNER LEONEL \\ São Carlos School of Engineering, Department of Structural Engineering, University of São Paulo, Brazil
}

\begin{abstract}
This study presents a numerical scheme for the fatigue crack growth modelling in plane nonhomogeneous structural systems. The structural domain is assumed as composed of piecewise homogeneous isotropic materials. The high-cycle fatigue case is assumed. Consequently, the linear elastic fracture mechanics theory is utilized. The mechanical behaviour is represented by the dual boundary element method (DBEM), in which singular and hypersingular integral equations are applied. The DBEM is an efficient and robust numerical technique for crack propagation analyses, especially due to the non-requirement of the domain mesh. This aspect enables the accurate representation of the elastic fields around the crack tip and simplifies the remeshing process during the crack propagation. The singular integrals are evaluated through the singularity subtraction method. Third degree polynomial transformation is utilized for improving the accuracy of near-singular integrals. The interface between adjacent materials is assumed as perfectly bonded and the sub-region BEM approach is utilized for representing the nonhomogeneous bodies. The Paris' law is utilized for determining the crack growth rate. The stress intensity factors are assessed by the J-integral and the maximum circumferential stress theory is adopted for defining the crack propagation angle and the range of equivalent stress intensity factor. The structural life is determined by a special scheme, which is based on discrete crack increments. One application is presented to illustrate the efficiency of the proposed model in simulating the fatigue crack growth in multiple cracked structures.
\end{abstract}

Keywords: fatigue, multiple crack growth, coalescence, dual boundary element method, nonhomogeneous domains.

\section{INTRODUCTION}

The application of materials with dissimilar mechanical properties in nonhomogeneous structural systems has recently increased in several engineering fields. In this type of typology, the best mechanical feature of each material is explored, resulting in better structural performance. However, the materials contain inherent flaws at some level of its microstructure. Thus, under oscillating stresses, such flaws may join to form a crack, which in turn may grow and lead to the mechanical failure. This phenomenon is known as fatigue and it is a main concern regarding structures subjected to cyclic loading. Fatigue failure may occur even if the stresses along the material are below its static tensile strength. Therefore, this type of collapse is sudden, i.e., without previous notice, and usually catastrophic. Hence, the proposition of models to analyse accurately fatigue crack growth problems and to predict the structural life of nonhomogeneous structures is a priority in engineering design. Moreover, these models must consider the influence of different material properties during the crack propagation through the structure and the interaction between cracks to represent reliably general and complex problems.

The mechanical analysis of cracked nonhomogeneous structures has been performed experimentally [1], [2]. However, the robust mechanical modelling of this problem requires numerical methods because of the complexity involved into the material discontinuities description in addition to the boundary conditions. In spite of domain mesh methods are utilized in this field, such as the finite element method [3] and the extended finite element 
method [4], the boundary element method (BEM) is the proper numerical method for handling such mechanical problem. The BEM was accurately utilized for assessing the stress intensity factors (SIF) in cracked nonhomogeneous bodies by [5], [6]. Afterwards, improved BEM models, based on the dual BEM (DBEM) formulation, enabled the simulation of multiple crack propagation and coalescence phenomena [7], [8]. However, the studies considering multiple crack growth in nonhomogeneous bodies are scarce in the literature, which justifies the development of the present research.

In the present study, a numerical model to simulate the fatigue crack growth in multiple cracked nonhomogeneous bodies is presented. The DBEM is utilized to assess the structural mechanical behaviour. The material of each subdomain, which compose the nonhomogeneous structural system, is considered as homogeneous and isotropic. The subregion formulation of the BEM is applied for coupling the entire domain. The interface between adjacent materials is assumed as perfectly bonded and delamination is not addressed. The proposed approach is capable for simulating the coalescence phenomenon and the crack intersection with the external boundary and with the materials interfaces. The Paris' law is utilized to obtain the crack growth rates and the SIF are evaluated through the J-integral technique. The crack propagation angle is determined by the maximum circumferential stress criterion. An especial methodology is proposed for defining the structural life, which accounts for discrete crack growth increments. One application is utilized to demonstrate the robustness of the proposed numerical approach.

\section{THE DUAL BOUNDARY ELEMENT METHOD}

Consider a plane, homogeneous and linear elastic domain $\Omega$ with boundary $\Gamma$ subjected to static loads. Using the Betti's reciprocal work theorem and disregarding the body forces, the displacements $u_{i}$ at an internal point $s$ of this domain are obtained from the displacements $u_{j}$ and tractions $p_{j}$ at the boundary as follows:

$$
u_{i}(s)=\int_{\Gamma} U_{i j}^{*}(s, f) p_{j}(f) d \Gamma-\int_{\Gamma} P_{i j}^{*}(s, f) u_{j}(f) d \Gamma,
$$

where $U_{i j}^{*}$ and $P_{i j}^{*}$ are, respectively, the displacement and traction fundamental solutions, which are functions of the distance $r$ between the source point $s$ and the field point $f$ at the boundary.

By differentiating eqn (1) with respect to the internal point, then using the straindisplacement relation and finally applying the Hooke's law, the equation that define the stress components $\sigma_{j k}$ at the point $s$ is obtained, which is given by:

$$
\sigma_{j k}(s)=\int_{\Gamma} D_{i j k}^{*}(s, f) p_{i}(f) d \Gamma-\int_{\Gamma} S_{i j k}^{*}(s, f) u_{i}(f) d \Gamma,
$$

where $D_{i j k}^{*}$ and $S_{i j k}^{*}$ are fundamental solutions obtained from the derivatives of $U_{i j}^{*}$ and $P_{i j}^{*}$, respectively, which are also functions of $r$.

Eqns (1) and (2) contain regular integrals. Moreover, such equations enable the computation of the elastic fields at the internal points because the displacement and traction components at the boundary are known. To define such components, i.e. at the boundary, the source point $s$ is taken to the boundary. By performing this limit process with eqn (1), the displacement boundary integral equation (DBIE) is determined as follows:

$$
c_{i j}(s) u_{j}(s)+c_{i j}(\bar{s}) u_{j}(\bar{s})+\int_{\Gamma} P_{i j}^{*}(s, f) u_{j}(f) d \Gamma=\int_{\Gamma} U_{i j}^{*}(s, f) p_{j}(f) d \Gamma,
$$


where $c_{i j}$ is a coefficient resulting from the limit process on the integral containing $P_{i j}^{*}$, which is equal to $\delta_{i j} / 2$ for $s$ at the smooth boundaries, being $\delta_{i j}$ the Kronecker delta. The point $\bar{s}$ refers to a potential source point at the same position of $s$ but at different surface. This occurs, for instance, for source points at opposite crack surfaces. When $s$ does not have a corresponding point, the $\bar{s}$ term in eqn (3) is nil.

Similar limit process is performed with eqn (2). Then, using the Cauchy's formula, the traction boundary integral equation (TBIE) is obtained. For the source point at smooth boundaries, the TBIE is given by:

$$
\frac{1}{2}\left[p_{j}(s)-p_{j}(\bar{s})\right]+n_{k}(s) \int_{\Gamma} S_{i j k}^{*}(s, f) u_{i}(f) d \Gamma=n_{k}(s) \int_{\Gamma} D_{i j k}^{*}(s, f) p_{i}(f) d \Gamma,
$$

where $n_{k}$ are the components of the normal versor to the boundary at the source point.

Eqns (3) and (4) contain improper integrals because the fundamental solutions present different types of singularity as $r$ approaches zero. The weak singularity, of order $\ln (r)$, occurs in $U_{i j}^{*}$; the strong singularity, of order $1 / r$, is observed in both $P_{i j}^{*}$ and $D_{i j k}^{*}$; and the hypersingularity, of order $1 / r^{2}$, occurs in $S_{i j k}^{*}$. Therefore, the kernels with strong singularity and hypersingularity must be evaluated in the sense of Cauchy principal value and Hadamard finite part, respectively.

In this study, the algebraic system of equations is obtained via DBEM by the numerical evaluation of eqns (3) and (4) [9], [10]. The boundary $\Gamma$ is subdivided into isoparametric elements with polynomial approximation. High order boundary elements are available. The boundary elements contain the collocation points, which are used as source points of the integral equations. The TBIE is applied to the discretization along one of the crack surfaces, whereas the DBIE is applied to the opposite crack surface and to the external boundary.

The numerical integration is performed with the standard Gauss-Legendre quadrature when the integrated element is far from the source point, because such integrals are regular. The Telles' third degree polynomial transformation [11] is utilized when integrating near singular elements. To integrate the singular elements, i.e. elements that contain the source point, the singularity subtraction method is utilized. Discontinuous or semi-discontinuous elements are utilized for ensuring smooth boundary at collocations points and for enforcing the boundary conditions discontinuity between adjacent elements. In those elements, both or just one of the collocation points at the end are placed inside the element, respectively. Moreover, the existence of the Hadamard finite part in the TBIE requires the continuity of the displacement derivatives at the collocation points, which is guaranteed with discontinuous elements. Therefore, this type of element is also utilized to model the crack surfaces.

In the proposed model, the nonhomogeneous structural system is composed of piecewise homogeneous materials, which are interconnected by interfaces. To model this mechanical condition, the sub-region BEM technique is utilized. Thus, each subdomain is composed exclusively of one material. Hence, the global mechanical problem is assembled by enforcing compatibility of displacements and equilibrium of forces along the adjacent subdomains.

\section{LINEAR ELASTIC FRACTURE MECHANICS}

The Linear Elastic Fracture Mechanics (LEFM) has been widely applied in fatigue modelling. Particularly, for determining the crack growth rate under oscillatory loading. This theory assumes that the inelastic process zone ahead of the crack tip is small in comparison 
with the crack length or even with other dimensions of the cracked body. Such behaviour resembles high-cycle fatigue problems, in which the strains developed within the structure are predominantly elastic. According to the LEFM theory, the stress field around the crack tip in a homogeneous material develops a singularity of order $r^{-1 / 2}$ as $r$ approaches zero. In addition, as for cracks near the interface, the stress field can have others orders of singularity [12]. Therefore, the use of the stress components in this region for analysing crack stability becomes compromised. Alternatively, the so-called stress intensity factors (SIF) are used to assess the crack stability for enabling fully description of the stress field near the crack tip. In plane problems, two values of SIF associated with the basic modes of fracture are defined: $K_{I}$, related to opening mode, or mode I, and $K_{I I}$, related to the sliding mode, or mode II.

In the present study, the fatigue model utilized for assessing the structural life is the Paris' law [13]. It states that the crack grows as a function of the amount of load cycle as follows:

$$
\frac{d a}{d N}=C(\Delta K)^{m}
$$

where $a$ is the crack length, $N$ indicates the amount of load cycles, $C$ and $m$ represent the material parameters and $\Delta K$ is the range of effective stress intensity factors.

Here, $\Delta K$ is assumed as equal to the difference between the maximum and the minimum values of the equivalent SIF, or $K_{e q}$, during one load cycle. The value of $K_{e q}$ is given by a combination of $K_{I}$ and $K_{I I}$ obtained by a desired criterion, commonly based on stress or energy methods. Several approaches have been introduced in the literature, e.g., the maximum circumferential stress criterion [14], the maximum energy release rate criterion [15] and minimum strain energy density criterion [16]. In this study, the maximum circumferential stress criterion is utilized. This criterion assumes that the crack grows along the direction $\theta_{p}$, which is perpendicular to the maximum circumferential stress near the crack tip. Thus, $\theta_{p}$ is defined as follows:

$$
\theta_{p}=\sin ^{-1}\left(\frac{K_{I} K_{I I}-3 K_{I I} \sqrt{8 K_{I I}^{2}+K_{I}^{2}}}{9 K_{I I}^{2}+K_{I}^{2}}\right) .
$$

With $\theta_{p}$ value defined, the value of $K_{e q}$ is assessed as follows:

$$
K_{e q}=\cos \left(\frac{\theta_{p}}{2}\right)\left[K_{I} \cos ^{2}\left(\frac{\theta_{p}}{2}\right)-\frac{3}{2} K_{I I} \sin \left(\theta_{p}\right)\right] .
$$

In this study, the SIF values for modes I and II are obtained with the path-independent Jintegral proposed by Rice and Sih [17]. This integral is evaluated along a path $\Gamma_{J}$ enclosing the crack tip being expressed by:

$$
J=\int_{\Gamma_{J}}\left(W n_{1}-p_{j} u_{j, 1}\right) d \Gamma
$$

where $W$ is the strain energy density given by $\int_{0}^{\varepsilon_{i j}} \sigma_{i j} d \varepsilon_{i j}, p_{j}$ are the tractions along $\Gamma_{J}$ obtained by $\sigma_{i j} n_{i}, u_{j}$ are the displacements along $\Gamma_{J}$ and $n_{i}$ are the components of the normal versor to the path. 


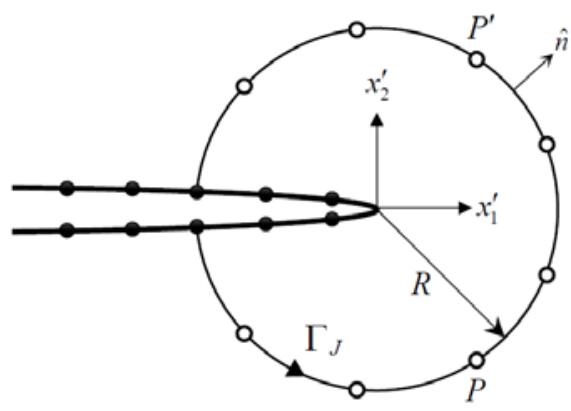

- Collocation point o Internal point

Figure 1: Path used to evaluate the J-integral.

In the proposed model, the path $\Gamma_{J}$ is assumed as circular and centred at the crack tip. It starts at a collocation point of the BEM mesh on one crack surface and finishes at the corresponding point on the opposite surface as illustrated in Fig. 1. Moreover, the integration path must be positioned inside the piecewise homogeneous material and cannot cross any other crack tip. To satisfy such conditions, a simpler automatic scheme is utilized to adjust the length of the path radius, which accounts for the intersection distances.

To evaluate the J-integral numerically in the context of the DBEM, a set of internal points is symmetrically positioned at the crack axis along $\Gamma_{J}$. Because the problem is analysed via DBEM, the displacements and stresses of the internal points are obtained into a postprocessing phase. For this purpose, the discretized forms of eqns (1) and (2) are applied. Then, the displacement vector and the stress state of the internal points are rotated to the coordinate system oriented according to the crack tip, as shown in Fig. 1. Here, to calculate $K_{I}$ and $K_{I I}$ separately from the J-integral in a mixed-mode problem, the mode decoupling technique proposed by [18] is utilized. In this approach, the displacements and stresses of two symmetric points $P$ and $P^{\prime}$ are decomposed into their symmetric and antisymmetric parts. By adopting this procedure, the J-integral presented in eqn (8) is also decoupled into a sum of two terms, $J^{I}$ and $J^{I I}$, with general expression given by:

$$
J^{M}=\int_{\Gamma_{J}}\left(W^{M} n_{1}-p_{j}^{M} u_{j, 1}^{M}\right) d \Gamma,
$$

where $M=I, I I$ represents the fracture mode. The mode I or mode II are considered if the symmetric or antisymmetric parts of the elastic fields is accounted in eqn (9), respectively.

By discretizing $\Gamma_{J}$ into a set of elements that contain the points defined along the path and by adopting a polynomial approximation for displacements and stresses over the element, the integral in eqn (9) can be evaluated numerically using the Gauss-Legendre quadrature. With $J^{I}$ and $J^{I I}$, the SIF values are determined by:

$$
\begin{gathered}
K_{I}=\sqrt{E^{*} J^{I},} \\
\left|K_{I I}\right|=\sqrt{E^{*} J^{I I}},
\end{gathered}
$$


where $E^{*}=E$ for plane stress or $E^{*}=E /\left(1-v^{2}\right)$ for plane strain, with $E$ and $v$ being the Young's modulus and Poisson's ratio, respectively.

It is worth mentioning that the value of $K_{I}$ is always positive and is assessed directly from eqn (10). On the other hand, the $K_{I I}$ value from eqn (11) gives only its magnitude, because both positive and negative values are allowed. The sign is positive if the displacement about $x_{1}^{\prime}$ for the top crack face is higher than for the bottom crack face, and it is negative otherwise.

Another relevant quantity to determine in fatigue problems is the structural life, i.e., the amount of load cycles until the failure. The failure is usually characterized by a high crack growth rate or by a macro crack that develops within the body, compromising its structural integrity. In the present study, the amount of load cycles $\Delta N_{i+1}$ required to a crack tip to grow from a certain length $a_{i}$ to a length $a_{i+1}$ is obtained from the integration of the Paris' law as follows:

$$
\Delta N_{i+1}=\int_{a_{i}}^{a_{i+1}} \frac{d a}{C(\Delta K)^{m}} .
$$

The $\Delta K$ value depends on the crack length $a$. Assuming sufficient small crack length increments $\Delta a=a_{i+1}-a_{i}, \Delta K$ can be written as a linear function of $a$ according to:

$$
\Delta K(a)=\left(\Delta K_{i+1}-\Delta K_{i}\right) \frac{\left(a-a_{i}\right)}{\Delta a}+\Delta K_{i},
$$

where $\Delta K_{i+1}$ and $\Delta K_{i}$ correspond to the values of $\Delta K$ at the crack tip evaluated to the lengths $a_{i+1}$ and $a_{i}$, respectively.

Substituting eqn (13) into eqn (12) and developing it algebraically, the expression to evaluate the increment in the amount of load cycles is obtained as follows:

$$
\Delta N_{i+1}=\frac{\Delta a\left(\Delta K_{i}^{1-m}-\Delta K_{i+1}^{1-m}\right)}{C(m-1)\left(\Delta K_{i+1}-\Delta K_{i}\right)} .
$$

When the crack tip crosses the structural boundary or another crack at the configuration $i+1$, it becomes inactive and the value of $\Delta K_{i+1}$ cannot be computed. In this case, only the value of $\Delta K_{i}$ is used to estimate $\Delta N_{i+1}$ by applying the discrete form of Paris' law as follows:

$$
\Delta N_{i+1}=\frac{\Delta a}{C\left(\Delta K_{i}\right)^{m}} .
$$

Moreover, eqn (14) is only valid if the crack tip remains in the same subdomain. Otherwise $\Delta N_{i+1}$ is evaluated by using the discrete form of Paris' law considering each crack increment along a subdomain separately.

Suppose the situation described in Fig. 2(b), where a crack tip initially at material 1 grows $\Delta a_{1}$ in material 1 and $\Delta a_{2}$ in material 2. Taking the corresponding value of $\Delta K$ as the one obtained when the tip is at same material, $\Delta N_{i+1}$ is given by: 


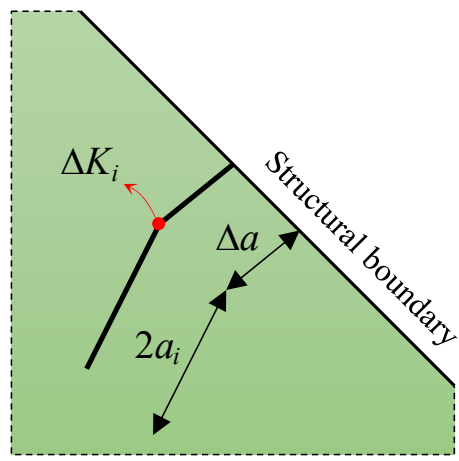

(a)

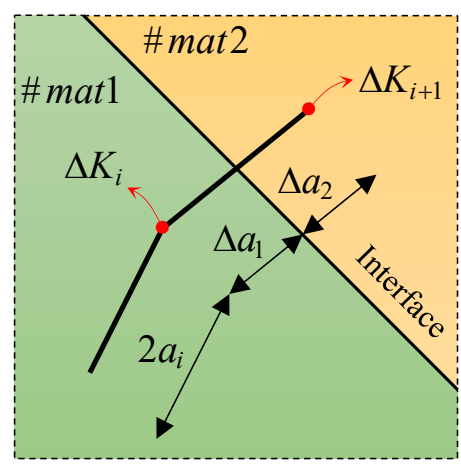

(b)

Figure 2: Crack growth. (a) To the structural boundary; and (b) Between subdomains.

$$
\Delta N_{i+1}=\frac{\Delta a_{1}}{C_{1}\left(\Delta K_{i}\right)^{m_{1}}}+\frac{\Delta a_{2}}{C_{2}\left(\Delta K_{i+1}\right)^{m_{2}}} .
$$

In a problem with multiple cracks, the value of $\Delta N_{i+1}$ is evaluated with eqns (14), (15) or (16) considering the crack tip with the highest growth rate at the configuration $i$.

\section{THE CRACK GROWTH SCHEME}

The solution process of the fatigue crack growth problem in a nonhomogeneous domain starts with the evaluation of the classical $\mathbf{H}$ and $\mathbf{G}$ matrices for the entire boundary element discretization. Such matrices are composed of the kernels presented in eqns (3) and (4). Thus, the DBEM and the sub-region BEM technique are utilized. Then, for each load phase that constitute one load cycle, one response to the boundary values is obtained by applying the corresponding boundary conditions and by solving the algebraic system of equations. The SIF values are also computed for the crack tips at each load phase in a post-processing stage using the J-integral technique. After all load phases are analysed, $\Delta K$ is evaluated for each tip by the difference between the maximum and minimum values of $K_{e q}$ determined into one load cycle. A given crack tip grow if the respective value of $\Delta K$ is higher than the threshold SIF range $\Delta K_{t h}$ defined for the material. The propagation angle of the growing crack is evaluated as the weighted average of the values of $\theta_{p}$ defined for all load phases adopting the corresponding $K_{e q}$ as weights, i.e.:

$$
\theta_{p}=\frac{\sum_{j}^{n l p} \theta_{p}^{(j)} K_{e q}^{(j)}}{\sum_{j}^{n l p} K_{e q}^{(j)}},
$$

in which $n l p$ is the amount of load phases during one load cycle and $\theta_{p}^{(j)}$ and $K_{e q}^{(j)}$ are, respectively, the propagation angle and the equivalent SIF for the $j$-th load phase defined by eqns (6) and (7). 
Along this direction, an increment at the crack length $\Delta L_{c}$ is defined as follows:

$$
\Delta L_{c}=\Delta L \frac{C_{s d} \Delta K^{m_{s d}}}{[d a / d N]_{\max }} .
$$

where $\Delta L$ is the standard crack length increment defined in the analysis, $C_{s d}$ and $m_{s d}$ are the parameters of Paris' law for the subdomain that contains the considered tip and $[d a / d N]_{\max }$ is the highest value of the crack growth rate observed during one load cycle.

From eqn (18), one notices that in a problem involving multiple cracks, some cracks can grow faster than others with smaller growth rate. This may imply in small length increments for the slower growing cracks. In this situation, some collocation points may be very close to other, compromising the accuracy of the DBEM solution. To avoid numerical issues, a virtual crack extension scheme similar to that proposed by [8] is applied. In this strategy, a crack tip propagates only if the following conditions are satisfied: a specified ratio between the lengths of adjacent elements must not be exceeded; and the crack length increment must be higher than a given ratio of $\Delta L$. If these conditions are not observed, the crack tip is not extended and the corresponding $\Delta L_{c}$ defined at that configuration is accumulated as a virtual length. In the course of the analysis, if the total virtual length exceeds both growing condition, then the crack grows with the accumulated length. This scheme certainly introduces approximations in the analysis. However, because the lengths involved in this procedure are small, it does not affect significantly the solution accuracy.

When the crack tips propagate, new collocation points are positioned along the path to discretize the new crack faces as illustrated in Fig. 3. To each collocation point, two additional degrees of freedom in displacements and tractions are created. Therefore, the corresponding influence coefficients are added at $\mathbf{H}$ and $\mathbf{G}$ matrices using the DBEM formulation. In addition, the new crack increments may intersect a pre-existing element in the boundary mesh. In this case, the intersected element is modified and a new element is created, Fig. 3(b). The influence coefficients related to the modified element are changed, whereas the influence coefficients related to the new element are added at $\mathbf{H}$ and $\mathbf{G}$ matrices. The Fig. 3(c) represents the changes of the BEM matrices with additions and modifications of elements on the mesh.

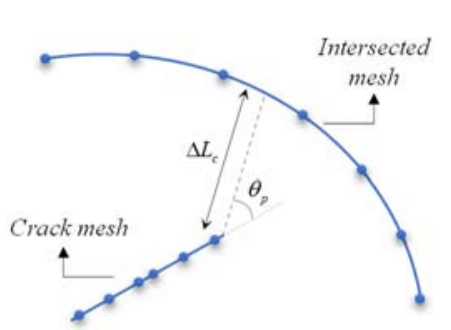

(a)

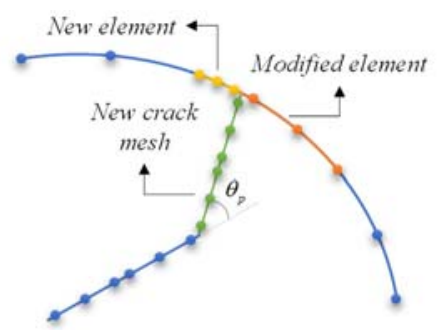

(b)

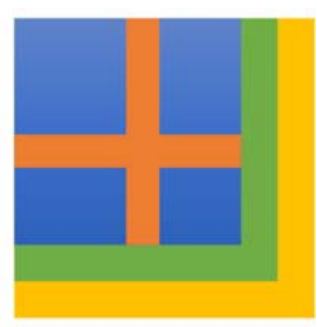

(c)

Figure 3: Representation of: (a) Initial mesh composed of quadratic elements; (b) The remeshing procedure during the crack growth; (c) The changes in $\mathrm{H}$ and $\mathrm{G}$ matrices when elements are created and modified during crack propagation. 
After a new crack configuration is defined, the solution of the structure is repeated for each load phase. The amount of load cycles to obtain the new configuration from a previous one is evaluated with eqns (14), (15) or (16), according to the current position of the critical crack tip.

The fatigue failure of the structure is achieved when the growth rate for a given crack tip becomes large, usually in the order of millimetres per cycle. Moreover, if the maximum value of $K_{e q}$ during one load cycle exceeds the fracture toughness of the material $K_{I c}$, it is considered that unstable crack propagation occur and the structure fails by fracture.

\section{APPLICATION}

The nonhomogeneous structure analysed in this application is presented in Fig. 4. The left structural end is fixed along both directions and an oscillatory load $p$, with uniform magnitude ranging from 0 to $10 \mathrm{MPa}$, was applied along a half of the hole, as illustrated in Fig. 4. Ten cracks with average length equal to $10 \mathrm{~mm}$ were randomly distributed at each subdomain and two other cracks with fixed length equal to $10 \mathrm{~mm}$ were disposed at the hole boundary along the diagonal perpendicular to load direction. The following dimensions were considered: $L=400 \mathrm{~mm}, h=100 \mathrm{~mm}, r=40 \mathrm{~mm}, R=100 \mathrm{~mm}$ and $\alpha=\omega=45^{\circ}$.Plane stress conditions were assumed in this analysis. The Poisson's rates, $v$, and the Paris' law parameters were assumed the same for both materials, with $v_{1}=v_{2}=0.2, C_{1}=C_{2}=1.0 \cdot 10^{-10}$ and $m_{1}=m_{2}=2.75$, in which $d a / d N$ is given in $\mathrm{mm} / \mathrm{cycle}$ and $\Delta K_{e q}$ in $M P a \cdot \sqrt{\mathrm{mm}}$. Three different Young's moduli ratios were assumed. Such ratio is defined as $\beta=E_{1} / E_{2}$ and the following scenarios were considered: (a) $\beta=1$ (homogeneous domain); (b) $\beta=0.25$ and (c) $\beta=4$. For all these scenarios, $E_{1}$ was assumed equals to $10000 \mathrm{MPa}$.

The boundary element mesh was composed of quadratic elements. The structural boundary was discretized into 124 elements, whereas each crack was discretized into 4 elements. During the crack propagation, the maximum and minimum crack length increments were $5 \mathrm{~mm}$ and $1 \mathrm{~mm}$, respectively, and the maximum ratio between the lengths of adjacent crack elements was defined equal to 1.5. The Fig. 5 presents the deformed shapes for the final configuration accounting for the three scenarios analysed. The Fig. 5(a) illustrates the final configuration for $\beta=1$, which represents the homogeneous case. For the initial configuration, the cracks (1) and (2) were critical and developed the highest growth rates. Afterwards, the crack (3) became dominant, because it was at a region with the high stress level. This crack first grew towards and intersected the top edge of the structure. Then, it

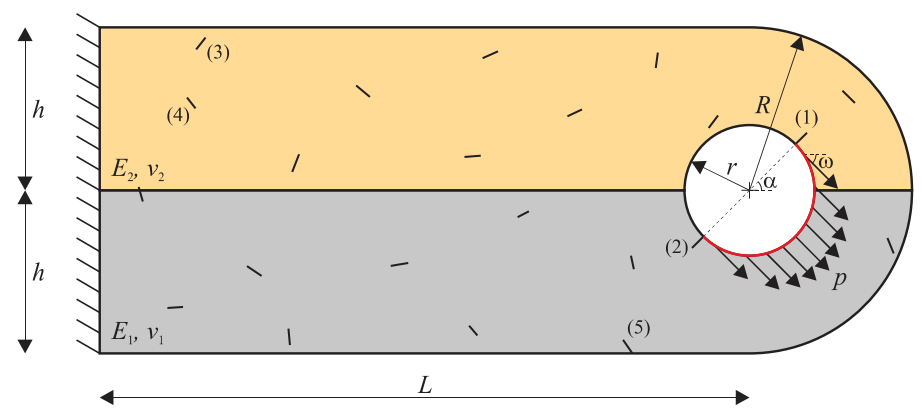

Figure 4: Nonhomogeneous structure with multiple cracks. 
propagated downwards, coalesced with crack (4) and crossed the interface between the materials. Finally, it intersected the bottom edge, finishing the analysis. The final deformed shape for $\beta=0.25$ is presented in Fig. 5(b). In this case, material 2 was subjected to the highest stress level, because it has the higher Young's modulus. Therefore, just the crack (1) was dominant in the beginning. In the course of the analysis, the crack (3) developed the highest growth rates and propagated through a path similar to that observed for $\beta=1$. Finally, the scenario of $\beta=4$ is considered and illustrated in Fig. 5(c). Because of material 1 is the stiffest, it developed higher stress levels than in the previous cases. In this scenario, crack (2) was dominant throughout the simulation and intersected the crack (5) in the end of the analysis.

The Fig. 6(a) presents the variation of $\Delta K_{e q}$ for the crack tip with the highest growth rate at each configuration with the cumulative crack length increment prescribed for the critical tips during the analysis. The curves for $\beta=1$ and $\beta=0.25$ show similar behaviours, because the critical crack tips at both models were the same. At the initial configuration, the maximum value of $\Delta K_{e q}$ for $\beta=0.25$ was higher than that determined for $\beta=1$ because of the higher stress level developed in material 2, as discussed before. A sudden decrease of $\Delta K_{e q}$ is observed when $\beta=0.25$ for a total crack length increment around $90 \mathrm{~mm}$. This represents the subdomain change of the critical tip to the material with lower stiffness. After the critical crack penetrated into material 1 , the values of $\Delta K_{e q}$ observed for both curves were similar, because the remained configurations were also similar.

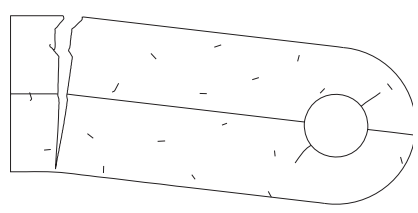

(a)

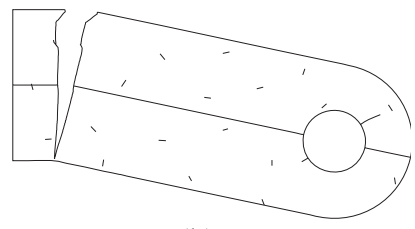

(b)

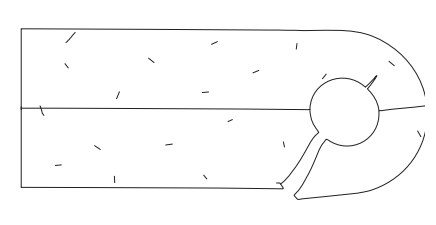

(c)

Figure 5: Deformed shapes of the final configurations. (a) $\beta=1$; (b) $\beta=0.25$; and (c) $\beta=4$. Nonhomogeneous structure with multiple cracks.

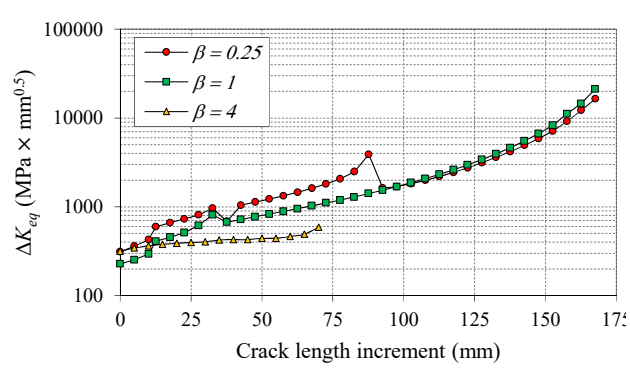

(a)

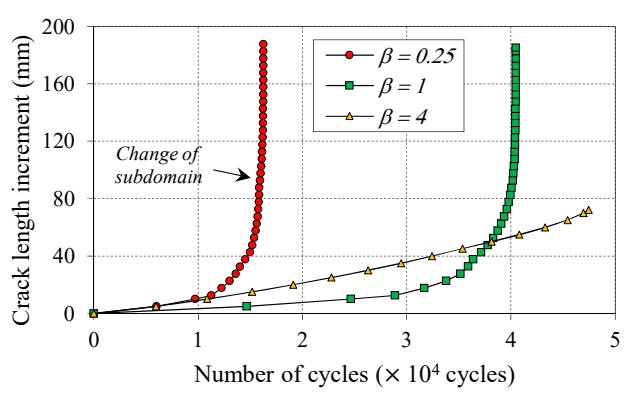

(b)

Figure 6: (a) $\Delta K_{e q}$ variation; and (b) Structural life. 
Other sudden changes in $\Delta K_{e q}$ is observed for both $\beta=0.25$ and $\beta=1$ cases. The first, around $10 \mathrm{~mm}$, indicates the intersection of one of the tips of crack (3) with the top edge of the structure, resulting into a raise of $\Delta K_{\text {eq }}$ value for its other tip at the following configuration. The second, around $40 \mathrm{~mm}$, represents the intersection between cracks (3) and (4) for $\beta=1$ and the shielding effect between the same cracks for $\beta=0.25$. In the latter situation, the cracks only coalesced in the next configuration. For $\beta=4$, the response for the initial configuration was the same as for $\beta=0.25$, because the critical crack in both cases was at the hole edge and in the stiffest material. Afterwards, the $\Delta K_{e q}$ values monotonically increased during the analysis, but remained lower than the previous cases.

The structural life predictions for the analysed cases are shown in Fig. 6(b). Because the same Paris' law parameters were assumed for all materials and cases, the curves behaviours are directly related to the $\Delta K_{e q}$ variation presented in Fig. 6(a). Therefore, it can be noticed that the higher the value of $\Delta K_{e q}$ the higher the crack growth rate and, consequently, the lower the structural life. The following structural lives were determined for the three cases: $16,000,40,000$ and 47,000 load cycles for $\beta=0.25, \beta=1$ and $\beta=4$, respectively.

This application demonstrated the robustness of the proposed model in handling fatigue crack propagation problems in multiple cracked nonhomogeneous domains. The simulations were able to efficiently represent the intersection of cracks with the external boundary, the interface between materials and other cracks. Moreover, the responses were stable and in accordance with the expected results.

\section{CONCLUSIONS}

In this study, a DBEM model was proposed to simulate the fatigue crack growth in plane nonhomogeneous domains considering the LEFM theory. The structures were assumed as composed of piecewise homogeneous and isotropic materials. Moreover, the interfaces between materials were assumed perfectly bonded.

The proposed model was capable to successfully represent the interaction between cracks in problems involving multiple cracked domains, including coalescence. The structural life was also efficiently predicted by the presented scheme. The DBEM model can be further extended to deal with delamination problems by considering the strength of the interface. In addition, anisotropic materials can be accounted to analyse other cases of practical importance.

\section{ACKNOWLEDGEMENT}

Sponsorship of this research project by the São Paulo State Foundation for Research (FAPESP), project number 2016/23649-0, is greatly appreciated.

\section{REFERENCES}

[1] Jiang, F., Kang, W., Zhao, K., Yan, C., Ye, L. \& Mai, Y.M., Experimental investigation on fatigue crack growth normal to a bimaterial interface. Structural Integrity and Fracture, eds A. Dyskin, H. Xiaozhi \& E. Sahouryeh, pp. 137-140, 2002.

[2] Kolednik, O., Predan, J. \& Fischer, F., Cracks in inhomogeneous materials: Comprehensive assessment using the configurational forces concept. Engineering Fracture Mechanics, 77(14), pp. 2698-2711, 2010.

DOI: 10.1016/j.engfracmech.2010.07.002. 
[3] Dias, M., Crack Propagation on Highly Heterogeneous Composite Materials. Technische Universiteit Eindhoven: Eindhoven, 2008.

[4] Yu, H., Sumigawa, T., Wu, L. \& Kitamura, T., Generalized domain-independent interaction integral for solving the stress intensity factors of nonhomogeneous materials. International Journal of Solids and Structures, 67-68, pp. 151-168, 2015. DOI: 10.1016/j.ijsolstr.2015.03.035.

[5] Ryoji, Y. \& Cho, S.B., Efficient boundary element analysis of stress intensity factors for interface cracks in dissimilar materials. Engineering Fracture Mechanics, 34(1), pp. 179-188, 1989. DOI: 10.1016/0013-7944(89)90251-8.

[6] Tullock, D., Reimanis, I., Graham, A. \& Petrocic, J., Deflection and penetration of cracks at an interface between two dissimilar materials. Acta Metallurgica et Materialia, 42(9), pp. 3245-3252, 1994. DOI: 10.1016/0956-7151(94)90422-7.

[7] Leonel, E. \& Venturini, W., Multiple random crack propagation using a boundary element formulation. Engineering Fracture Mechanics, 78(6), pp. 1077-1090, 2011. DOI: 10.1016/j.engfracmech.2010.11.012.

[8] Price, R. \& Trevelyan, J., Boundary element simulation of fatigue crack growth in multi-site damage. Engineering Analysis with Boundary Elements, 43, pp. 67-75, 2014. DOI: 10.1016/j.enganabound.2014.03.002.

[9] Hong, H.K. \& Chen, J.T., Derivations of integral equations of elasticity. Journal of Engineering Mechanics, 114(6), pp. 1028-1044, 1988. DOI: 10.1061/(asce)07339399(1988)114:6(1028).

[10] Portela, A., Aliabadi, M. \& Rooke, D., The dual boundary element method: Effective implementation for crack problems. International Journal for Numerical Methods in Engineering, 33(6), pp. 1269-1287, 1992. DOI: 10.1002/nme.1620330611.

[11] Telles, J., A self-adaptive co-ordinate transformation for efficient numerical evaluation of general boundary element integrals. International Journal for Numerical Methods in Engineering, 24(5), pp. 959-973, 1987. DOI: 10.1002/nme.1620240509.

[12] Zak, A. \& Williams, M., Crack point stress singularities at a bi-material interface. Journal of Applied Mechanics, 30(1), pp. 142-143, 1963. DOI: 10.1115/1.3630064.

[13] Paris, P., Gomez, M. \& Anderson, W., A rational analytic theory of fatigue. The Trend in Engineering, 13, pp. 9-14, 1961.

[14] Erdogan, F., Stress distribution in a nonhomogeneous elastic plane with cracks. Journal of Applied Mechanics, 30(2), pp. 232-236, 1963.

[15] Hussain, M., Pu, S. \& Underwood, J., Strain energy release rate for a crack under combined mode I and mode II. National Symposium on Fracture Mechanics, pp. 228, 1974.

[16] Sih, G., Strain-energy-density factor applied to mixed mode crack problems. International Journal of Fracture, 10(3), pp. 305-321, 1974.

DOI: $10.1007 / \mathrm{bf00035493.}$

[17] Rice, J. \& Sih, G., Plane problems of cracks in dissimilar media. Journal of Applied Mechanics, 32(2), pp. 418-423, 1965. DOI: 10.1115/1.3625816.

[18] Ishikawa, H., Kitagawa, H. \& Okamura, H., J integral of a mixed mode crack and its application. Mechanical Behaviour of Materials, pp. 447-455, 1980.

DOI: 10.1016/b978-1-4832-8414-9.50137-1. 